\title{
Dynamic Generation Method of Virtual Coal Mining Scene Based on 3D Entities Transformation and Combination
}

\author{
Shaojie Hou ${ }^{1,2, a, *}$, Xianzun Meng ${ }^{1, b}$ and Yuwei Zhang ${ }^{3, c}$ \\ ${ }^{1}$ Computer Center, Hebei University of Economics and Business, Shijiazhuang \\ 050061, China \\ ${ }^{2}$ Post-doctoral Scientific Research Workstation, Shanxi Coking Coal Group CO., \\ LTD, Taiyuan, Shanxi 030024, China \\ ${ }^{3}$ Shijiazhuang Vocational College of Finance Economics, Shijiazhuang 050061, \\ China \\ ashaojhou@163.com, ${ }^{b}$ mengxzun@163.com, ${ }^{c}$ zhangyuweix@163.com \\ * Corresponding Author
}

\begin{abstract}
In order to present the virtual scene of underground coal mining a dynamic and datadriven method for the virtual scene generation was proposed and its experimental software was developed. The data model of the software system is classified as two types that are the primary model and the display model. The system structure is designed as four layers from bottom to top, namely storage layer, disflay layer, combination layer and operation layer. Storage layer, the lovest layer and the data center of the system to store all the primary models, is a database containing some data tables, views, constrains, rules and storage processes. Displat laver as the second lower layer is to implement an essential data-driven algorithm to transform the selected primary models into display models through scallng, stretching, rotating or assembly operations. Combination layer focuses on the whole scene building business based on the operation of related display model such as visible setting, connecting, handling the shading and presenting special effects. Operation layer as the top layer is to respond to user's instructions such as free roaming and screen pickup. Finally, the software is tested in Qinan colliery of China. Results show that the proposed method is practicable and efficient to implement virtual coal mining scene.
\end{abstract}

Keywords: virtual reality; $3 D$ entity; dynamic generation; entity combination

\section{Introduction}

With the rapid growth of Chinese economy, the domestic coal output of China has soared up to 3.6 billion tons in 2015 from 1.8 billion tons in 2004. Meanwhile, the coal exploitation depth has exceeded $800 \mathrm{~m}$ in about $80 \%$ of Chinese underground collieries 122. The "three high and two low" phenomenon, namely high geo-stress, high gas, high inhomogeneous, low permeability and low coal mass strength, becomes more and more prominent, which increases the risk of coal and gas outburst, coal mine fire and gas explosion. In fact, hundreds of coal gas accidents with nearly one thousand casualties occurred annually in China in the recent 5 years. By this, research subjects for the coal gas control theory have attracted the extensive attention worldwide. However, different from other fields such as aerospace engineering, machinery manufacturing, and petrochemical engineering, the coal mining industry lacks the primary support of digitalization technology at present [3-5]. Having neither the integrated software for the coal gas tracking and data process nor the virtual scene rendering technology for the underground coal seam and its mining engineering, coal mine engineers are forced to 
overly depend on CAD drawing. It is insufficient to accurately master the process of coal mining and gas drainage, let alone to predict the potential risks.

As a rising technology, virtual reality technique integrates multiple research fields, such as computer graphics, multimedia, sensor application, human-computer interaction and network [6-8]. By using computer networks, related peripheral equipment and specific software systems, it generates a suppositional scene in which the operator can manipulate or control anything they want with an immersive feeling. With the rapid development of hardware performance, to present a virtual scene for underground coal mining becomes feasible. Actually related achievements have sprung up in the last years [9-10]. Specifically, Denby proposed a risk evaluation method for the running status of the whole colliery, which is based on the virtual reality system "Safe VR", "VROOM", "Truck Simulator" and "Truck Inspection", a series of software systems developed by AIMS Research Unit, University of Nottingham, Nottingham of United Kingdom [11-13]. Scholars of 5DT Co. (the fifth Dimension Technologies Company of United States) successfully built many virtual entities in their digital coal mining software, for example, coal cutter, coal mining face and coal seam, etc. In Australia, "InTouch", a virtual reality software developed by Earthworks, can shows clients the 3D virtual scene of terrain, ore body, pits, profile, buildings, electromechanical facilities, coal mining equipment, landscape and so on. Furthermore, another software system "Virtual Mine" was developed by CSIRO Co. in Australia by using data-driven technology, which can not only present us a virtual scene involving explorato y boreholes, $3 \mathrm{D}$ ore body, pits, etc. but also provide a simple and intuitive interface for the human-computer interaction. So in the "Virtual Mine" system, to simulate the process of coal rining process or earthquake becomes feasible. In China, Wang B.S Zhang R.X., Mao X.F. and Wang Y.H. respectively elaborated the latest achieyements of virtual coal mining, which inspired the enthusiasm of Chinese scholars to furtherelated study based on domestic collieries. Yang T.J. put forward a primary method for surface collieries to integrate virtual reality technology, including human-computer inter ction, vision modeling, scene rendering and virtual roaming [14].

In general, several adyanced countries have built a number of primary virtual reality software systems of coal mining, but they are not suitable for the coal gas control engineering. However, it's very necessary for those countries with high coal gas reserves such as China, Rassia, Pakistan and India, because amount of safe accidents with heavy casualties have happened in there in the last decade and various coal and gas control measures have been widely used in the coal mining process.

In this paper, a data-driyen virtual reality software system will be elaborated, which is constituted of three kinds of data and four business layers. The data include primary models, display models and scene, and the four business layers are separately storage layer, display layer, combination layer and operation layer. They cooperate with each other to generate the virtual coal mining scene based on the parameters entering, the $3 \mathrm{D}$ entities' transformation and combination.

\section{Regional Gas Control Technology of Single Outburst Coal Seam}

Nowadays many regional gas control measures are developed and widely used to prevent coal and gas outburst in China. Their essential approach is to reduce the gas reserves of coal seam though the drainage of coal gas prior to coal mining. Various approaches have been proved to be very effective, for example, surface boreholes, crosscoal-seam grid boreholes, along-coal-seam boreholes, cross-coal-seam boreholes combined with along-coal-seam boreholes, closed laneway, high location boreholes, etc. In them cross-coal-seam boreholes combined with along-coal-seam boreholes was extensively used in single coal seam because of its lower cost, shorter constructing period 
and better draining effect [15-18]. It mainly consists of eight steps as the following description $[19,20]$ :

Drive a floor rock laneway under the given coal seam about 20-30m;

Construct borehole-fields on the both sides of the floor rock laneway;

Construct upward cross-coal-seam boreholes in the borehole-fields, and their paths should traverse the coal seam at least $0.5 \mathrm{~m}$ and their bottom should form a regular grid cover the U-type strip-range astride the designed coal laneway;

Seal each borehole with a pipeline to connect into the global drainage system, by which the coal gas could be pre-drained and its reserves could be reduced. When the gas pressure or gas content is lower than the given critical value, the outburst dangerousness is eliminated;

After the strip-range coal mass is proved safe, miners start driving U-type coal laneways, including mechanical laneway, ventilating laneway and open-off cut;

Construct along-coal-seam boreholes in the coal laneways, which should cover the whole range of the coal mining face without blank zones;

Seal the along-coal-seam boreholes with a pipeline to connect into the global gas drainage system, by which the coal gas of the mining coal face could be pre-drained;

After gas pressure or gas content is proved lower than the critical yalue, start coal mining.

Above technical process can be described as the Figure 1.

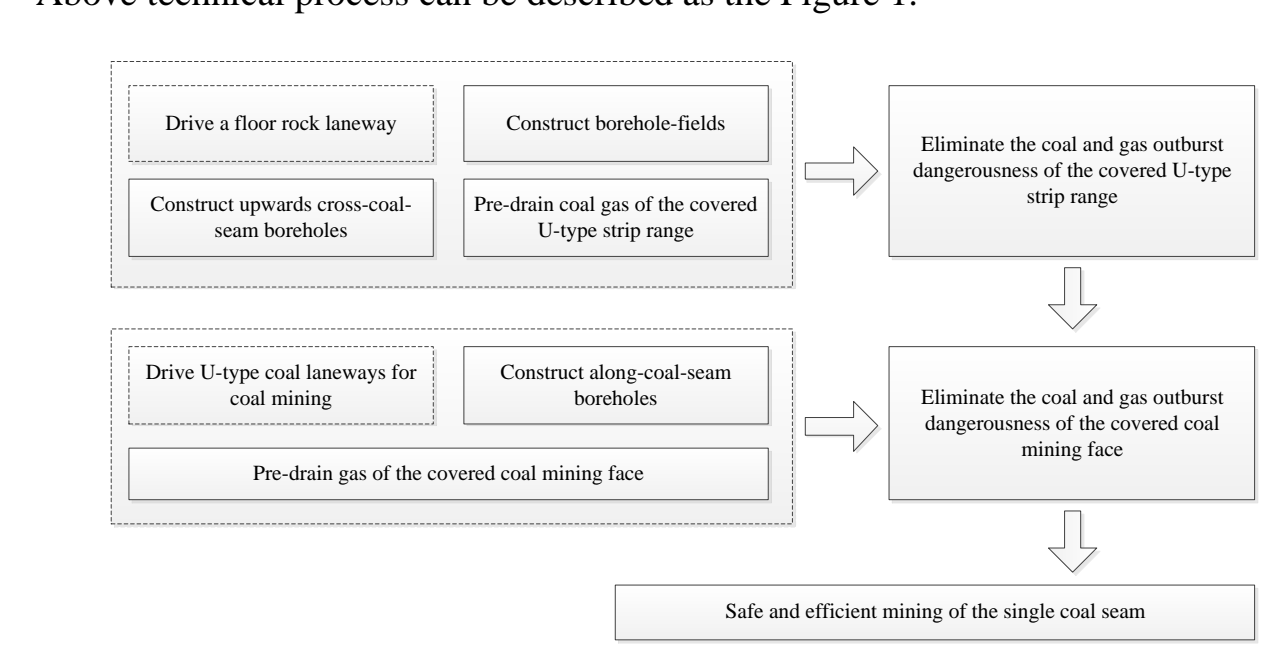

Figure 1. Process Of Regional Gas Pre-Drainage Technology Through Cross-Coal-Seam Boreholes Combined With Along-Coal-Seam Boreholes For The Single Coal Seam

\section{Prinary Models and Display Models}

From the perspective of virtual reality theory, the construction work of the coal gas pre-drainage through cross-coal-seam boreholes combined with along-coalseam boreholes as described above should essentially include the following components:

\subsection{Development of Primary Models}

After the disassembly of components involved in the construction work of coal gas predrainage, the more basic unit models, named as primary models, are gained as coal-seamunit model, rock-laneway-unit model, borehole-field-unit model, borehole-unit model and coal-laneway-unit model.

Coal-seam-unit Model 
Although many impurities and geologic structures exist in the large area of coal seams in practice such as coal gangue, stretch, fold and fault, coal mining faces are often pure and smooth because they are mostly set in a relatively little range of the coal seams. In order to simplify the architecture and improve computational efficiency, the coal-seamunit model is designed as a cuboid. It is one-unit-size, colored as RGB $(41,36,33)$ and developed in the 3D-Max software as shown in the Figure 2.

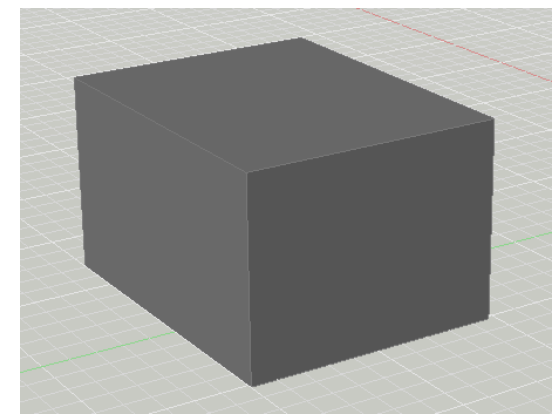

Figure 2. Coal-Seam-Unit Model

Rock-laneway-unit Model

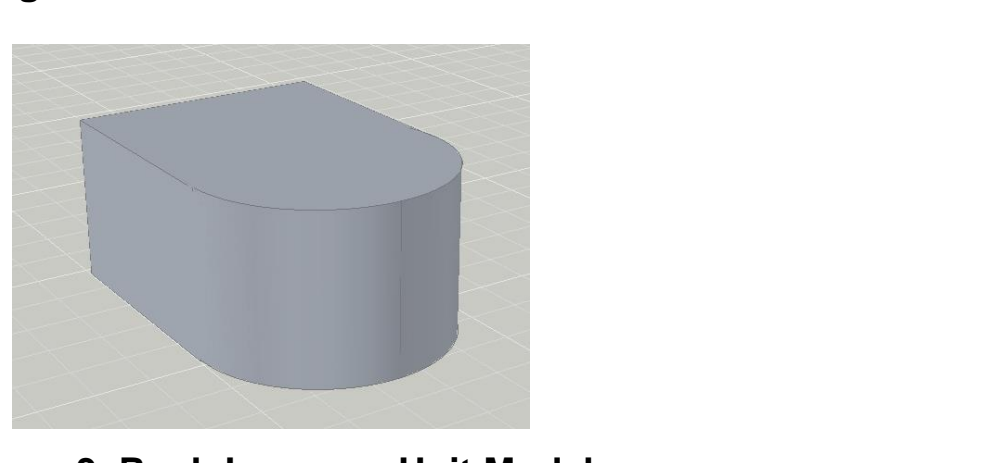

Figure 3. Rock-İaneway-Unit Model

To avoid the distortion and to defense the high stress of laneway's roof and sides, the rock laneway are often designed as arch in which the top arch's height is half of laneway's width. Setting the rock laneway's width as one-unit, its arch height as half-unit and its color as RGB $(192,192,192)$, the rock-laneway-unit model is developed as the Figure 3 shown.

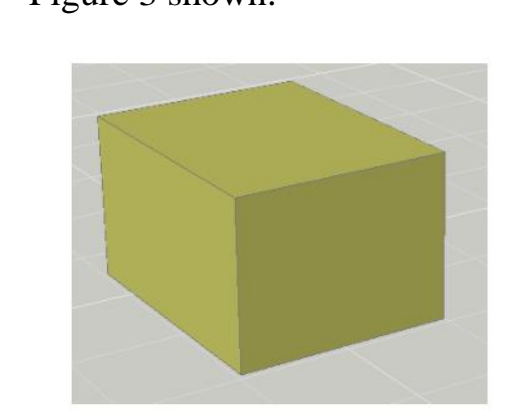

(a) Borehole-field-unit Model

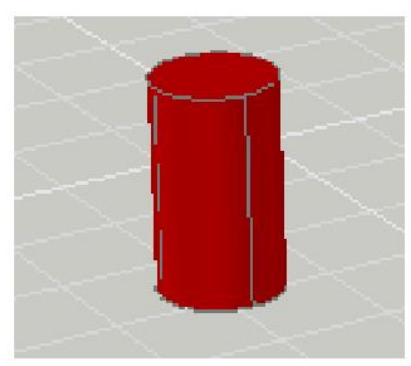

(b) Borehole-unit Model Model

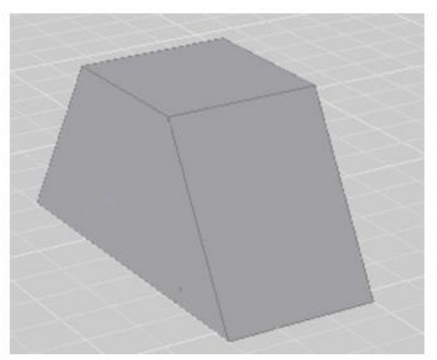

(c) Coal-laneway-unit

Figure 4. Other Unit Models

Other Unit Models

In the same way, the borehole-field-unit model is developed as a one-unit-size cube with RGB $(107,142,35)$ color; the borehole-unit model is developed as a cylinder with one-unit diameter and $\operatorname{RGB}(227,23,13)$ color; and the coal-laneway-unit model is 
developed as a structure with $\operatorname{RGB}(192,192,192)$ color, whose cross section is one isosceles trapezoid with 0.8 unit topline, 1.2 baseline and one-unit height. All they developed in the 3D-Max software as shown in the Figure 4.

\subsection{Build the Primary Library}

In practice, all the unit models are designed and developed in the most popular threedimensional modeling software Autodesk 3D Max 2012, which results in a series 3D Max document with some parameters. After this, the primary library for their storage is implemented in the relation database system Microsoft SQL Server 2008. Meanwhile, to cooperate with the development of our target software, some transformations for the file format and some data modeling of the unit models' parameters were done.

By the import function of 3D-Max software, all 3D unit models are transformed into the .FBX format files with some related parameters. Based on object-oriented theory, the .FBX format file are turned into the binary stream and stored as the image format field into the primary database, and the parameters are modeled into some data-fields with basic type, as shown in the Table 1.

\begin{tabular}{|c|c|c|c|}
\hline Number & Data-fields & Data Type & Descripti \\
\hline 1 & KeyX & $\operatorname{varchar}(50)$ & The identification of the unit model \\
\hline 2 & NameX & $\operatorname{varchar}(100)$ & The name of the unit model \\
\hline 3 & AssetNameX & $\operatorname{varchar}(100)$ & The name marked in the computer memory \\
\hline 4 & Object & image & The binary stream of the unit model \\
\hline 5 & WidthX & decimal $(18,2)$ & The initial Width of the unit model \\
\hline 6 & LengthX & $\operatorname{decimal}(18,2)$ & The initial length of the unit model \\
\hline 7 & HeightX & decimat $(18,2)$ & The initial length of the unit model \\
\hline
\end{tabular}

\subsection{Generation of Display Models}

The display models include coal seam, rock laneway, borehole field, cross-coal-seam borehole, U-type coal laneway and along-coal-seam borehole. Different from the unit models storing in the primary Vibrary, the display models aren't permanent stored. They are data-driyen and dynamically generated after a series transformation of the unit models.

In detail the primary libary is declared as a collection of memory objects, named as "primarymodels", wh ch is instantiated from the class "PrimaryModelDatas". Each element saved in the collection "primarymodels" represents one unit model. When the parameters of the Construction work are entered, the corresponding object " primarymodels will be forced to execute corresponding transformation such as scaling, stretching of rotating, and turn into the desired display models. The process is data-driven and automatically fulfilled through a series of the matrix transformation. Sometimes the display model is so complex that a number of assembly operations must be done such as U-type coal laneway, as shown in the Figure 5. 


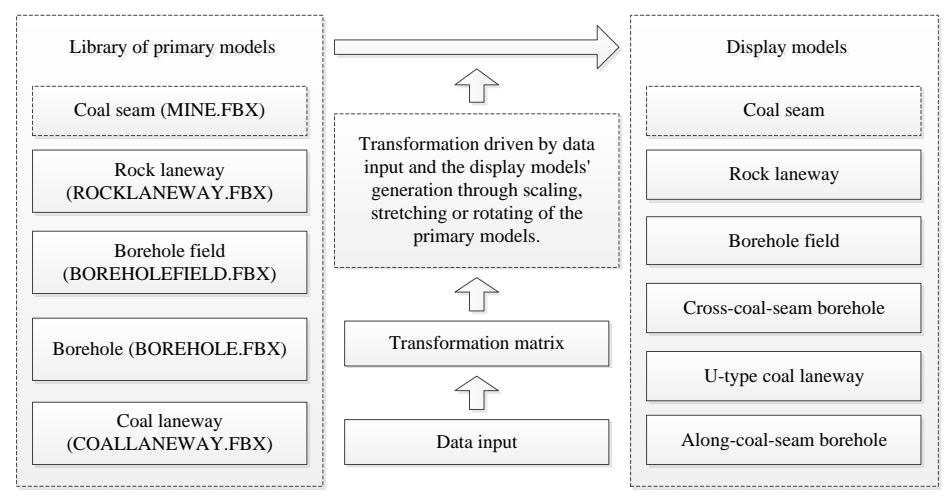

Figure 5. Generation of the Display Models

In the development, above algorithm is programmed by the $\mathrm{C \#}$ language based on .Net Framework 4.0 in the Microsoft Visual Studio 2010, an extensive used integrated development environment. Some code is given in the following Code 1.

\section{Code 1. Part Code of the Generation Process of Display Models}

// Declare the Collection Object of Primary Models

Models.PrimaryModelConfig.BasicModelDatas_primarymodels $=$ nul

// Declare the Collection Object of Display Models

Models.DisplayModelDatas_displaymodels = null;

// Fetch Unit Models from the Primary Library and Create object_primarymodels

this._primarymodels = new Models.BasicModelConfig_PrimaryModelDatas(Content);

// Create Object_displaymodels

this._displaymodels = new Models DísplayModelDatas();

// Part Code for the Generation of Display Models (Locate the Cross-seam-Borehole)

System.Data.DataSet dsgeneration = ModêlsDataSet._dsGeneration;

// Get the Parameters of Cross-seam-boreholes

System.Data.DataTable dtebs = dsgeneration.Tables["CBoreholes"];

// Get the Parameters of Borehole-fields

System.Data.DataTable dtcbfs = dsgeneration.Tables["CBoreholeFields"];

// Create Foreign Relation From Borehole-fields to Cross-seam-boreholes

System.Da a.DataRelation 2ofs_bs = new System.Data.DataRelation(

"r_bfs_bs ", dtcbrs.Columns["Id"], dt_zk.Columns["bf_Id"]);

dsgeneration.Relations.Add(r_bfs_bs);

// Locate the Crose-sean-Borehole

foreach (Systen.Data.DataRow $\mathrm{r}$ in dtcbs.Rows)

\{ // Get the Parameter of the Connected Borehole-field

System.Data.DataRow rcbfs = r.GetParentRow(r_bfs_bs);

f(rebfs == null) continue;

/ Do the Transformation

Models.DisplayModel dm=Model.CreateDefaultDisplayModel(r);

this._primarymodels.GetEntity(r).SetDefaultPosition();

this._primarymodels.GetEntity(r).DoScale(r);

this._primarymodels.GetEntity(r).DoStretch(r);

this._primarymodels.GetEntity(r).DoRotate(r);

\} 


\section{Development of the Virtual Reality System}

\subsection{System Architecture}

XNA was an integrated game studio express based on .Net framework, which was first issued by Microsoft Co. in August, 2006, and now has updated to version 4.0. Comparing with the traditional virtual technology DirectX, it provides a series of more integrated toolkits, interfaces and even one IDE. It can simplify the design of PC games and accelerate the development of virtual reality software.

Based on the XNA framework, a core class "VirtualMine" was developed by extending the global class "Game", in which the essential functions were implemented such as the system initialization, the import and release of $3 \mathrm{D}$ resources, the rendering and updating of 3D models.

When the "VirtualMine" class starts running, it performs the following five steps:

Perform the constructor "VirtualMine()" to create its memory object named as "VirtualMine";

Call the method "Initialize()" to define the designed system variables and global variables;

Call the method "LoadContent()" to import primary models, textures and background materials, and then to allocate memory for the roaming camera and database connection;

Enter a polling mode in which two important methods "Update()" and "Draw()" will be called alternatively and repeatedly to respond to any data update or users' interaction;

When user sends "EXIT" command, call the method "UnloadContent()" to release related resources and then exit system.

In above five steps, only the fourth step rans repeatedly and others run once, as the Figure 6 shows.I

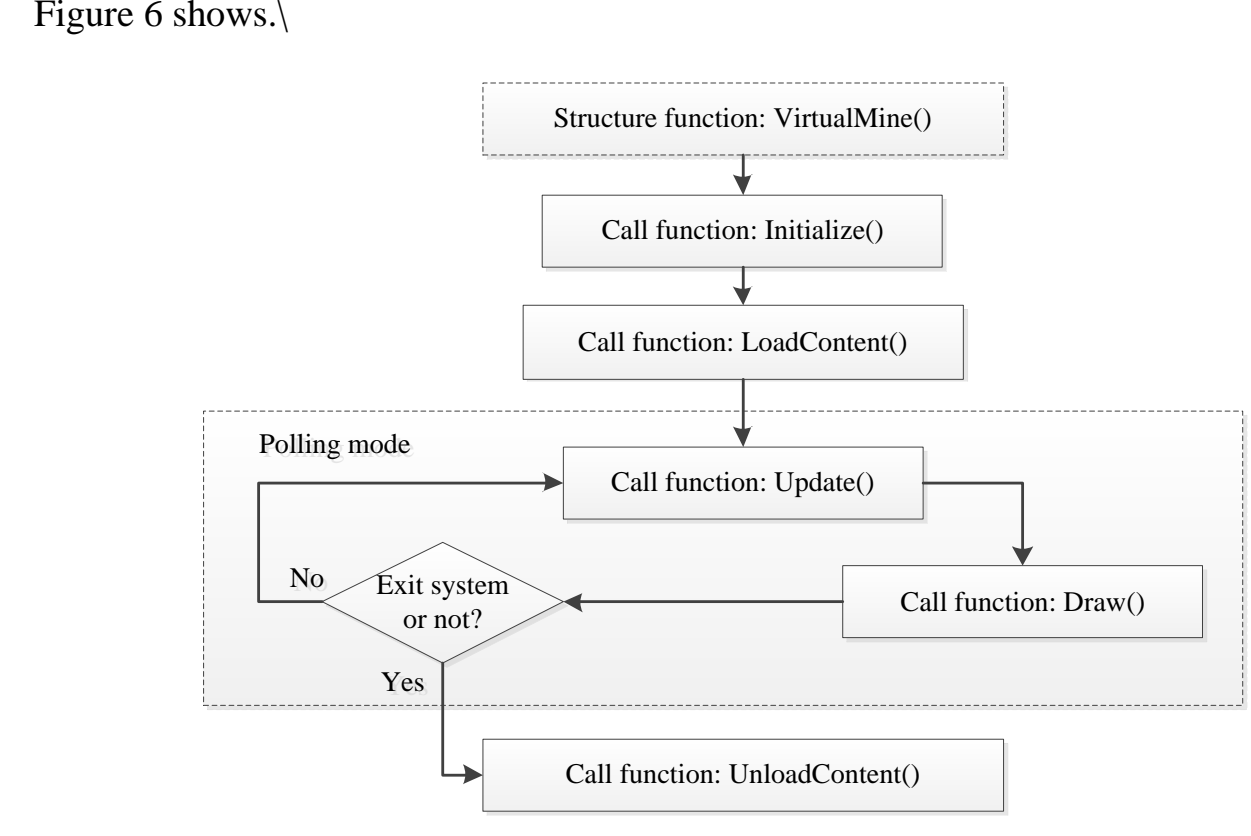

Figure 6. Architecture of the Virtual Reality System Based on XNA Framework 


\subsection{Four Business Layers}

In the research field of the software development, n-tier architecture was very popular in the recent years. In view of this thought, the complicated and bulky should be divided into several layers to simplify its designing, programming and maintaining process.

According to the demand analysis of the models' storage and transformation from primary models to display models, the rendering operations of the virtual scene was logically designed as four layers, namely storage layer, display layer, combination layer and operation layer from bottom to top, as the following description:

Storage layer. The storage layer is located at the bottom of the business logic and serves as the data center of the whole system. It is embodied as a SQL Server database including a series of tables, views, constrains, rules and storage processes, through which all the primary models and its parameters, all the data of coal gas control process and the system options are stored.

Display layer. The display layer is triggered by the data entering operation and generates the display models by primary models automatically. The generation process includes fetching related primary models from the storage layer and executing the scaling, stretching or rotating transformation on them. The simple display/model can be generated through the business of the display layer. But with regard to the complicated display models, only transformation operation cannot meet the denand. In this case, the display layer offers an assembly operation in further to build-up the final display models. For example, the U-type rock or coal laneway is consisted of three straight laneways is the case.

Combination layer. In view of the whole scene and the operators' view range, combination layer is to place the display models into the expected position and handle its' background, its shading or some specialeffects, and then finish the target virtual scene.

Operation layer. In practice, the virtual system should respond to user's operation immediately to improve users experience. The function will be implemented in the operation layer, mainly including free roaming and pickup click now.

\subsection{Free Roaming Technology}

In development of the virtual reality system, free roaming is often achieved by the transformation of global camera. Specifically, a view matrix must be created and maintained and whenever the rotating or offsetting movement of the scene happened, the corresponding matrix transformation must be done. In other words, the related function must be developed in the method "Draw()" for any rotation and offset movement [7,8].

Basically, in order to locate each entity into its correct place, a global right-handed spatial coordinate system is established in the scene as the Figure 7 shows.
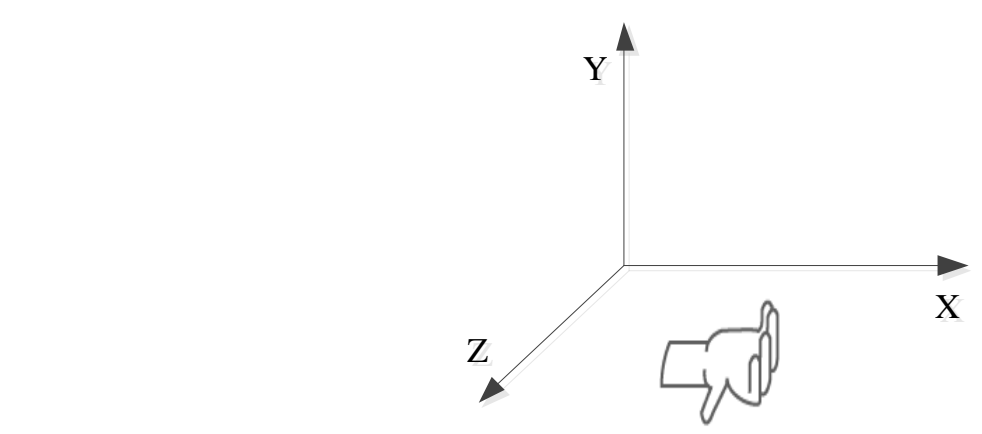

\section{Figure 7. Right-Handed Coordinate System}

When user wants to look up or down, the camera should rotate around the "Right" axis; when user wants to look left or right, the camera should rotate around the "Up" axis; but 
rotating around the "Forward" axis is forbidden because it means that the user would twist his neck left or right, which disaccustoms the view of first-person; when user wants to move the viewport such as go ahead, go back or go aside, the camera should make a corresponding transformation.

For this, two vectors were declared to describe the rotation quantity, named separately as "Right" and "Up". When user roams the scene, for example, operating mouse to go ahead, go back or move aside, the corresponding transformation of the view matrix will be executed by the following seven steps:

Reset vectors "Right" and "Up" as empty, and set camera anywhere;

Reset cursor to the center of screen, and save its position in an object "oldState" that is instantiated from the class "MouseState";

Get the offset of the mouse by comparing the new cursor's position "newState" with "oldState" after the user's operation;

Fill parameters of vectors "Right" and "Up" according the offset of mouse;

Call the method "Update()" to execute the corresponding matrix transformation based on the vectors "Right" and "Up";

Call method "Draw()" to paint the latest position of virtual entities;

Go to the first step and continue responding to the user's next ôperation

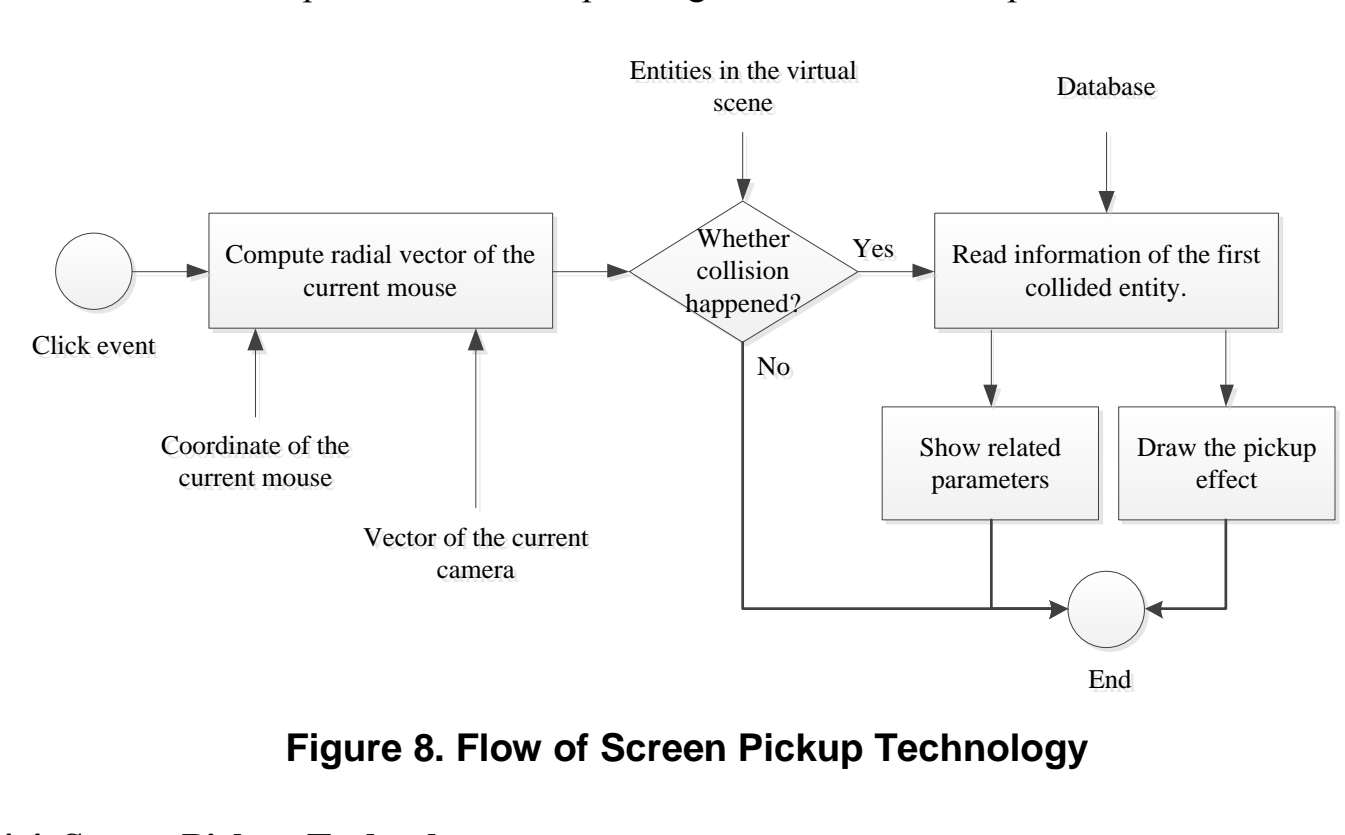

\subsection{Screen Pickup Technology}

In virtual reality system, screen pickup technology plays an important role to improve user's experience. It allows users to focus on some entities, and then pickup, highlight them as they roam. To develop this function, the cursor's planar position should be translated into a spatial outside-in radial and then interact with the display models. When the radial intersects with any entity, the entity becomes a candidate item to be picked up. If there are several candidate items, the first one should be selected considering the view habit of the first-person view. The process can be described as the following five steps:

Capture the click event of the mouse and trigger related programs for the pickup function;

Translate the planar position of click into a spatial outside-in radial which starts from the click point and along vector "Target" of the camera object;

Detect whether collision happens between the radial and the display models within the screen scope. When one or more than one entity, focus on the only or the first intersected display model, otherwise do nothing;

Read information of the focused entity and paint its tips on screen; 
Paint the preset effect of selected entity.

Above flow can be shown as the Figure 8.

\section{Application Effect}

Qinan colliery is located in Suzhou, Anhui province of China, where the No.716 coal mining face was selected as the experimental site for its representativeness of regional coal gas pre-drainage engineering. Two laneways, mechanical and ventilating laneways, are $985 \mathrm{~m}$ length, and the open-off cut is $145 \mathrm{~m}$ length.

With the detailed parameters being entered into the system as the Figure 9(a) shows, the virtual scene could show automatically, including coal seam and U-type laneways as the Figure 9(b) shows. In the same way, the parameters entering of borehole-fields, crosscoal-seam boreholes and along-coal-seam boreholes triggered their virtual entities show automatically as the Figure 9(c) shows. During user roams, any selected entity is would be highlighted and its description would be shown on the screen as the Figure 9(d) shows.
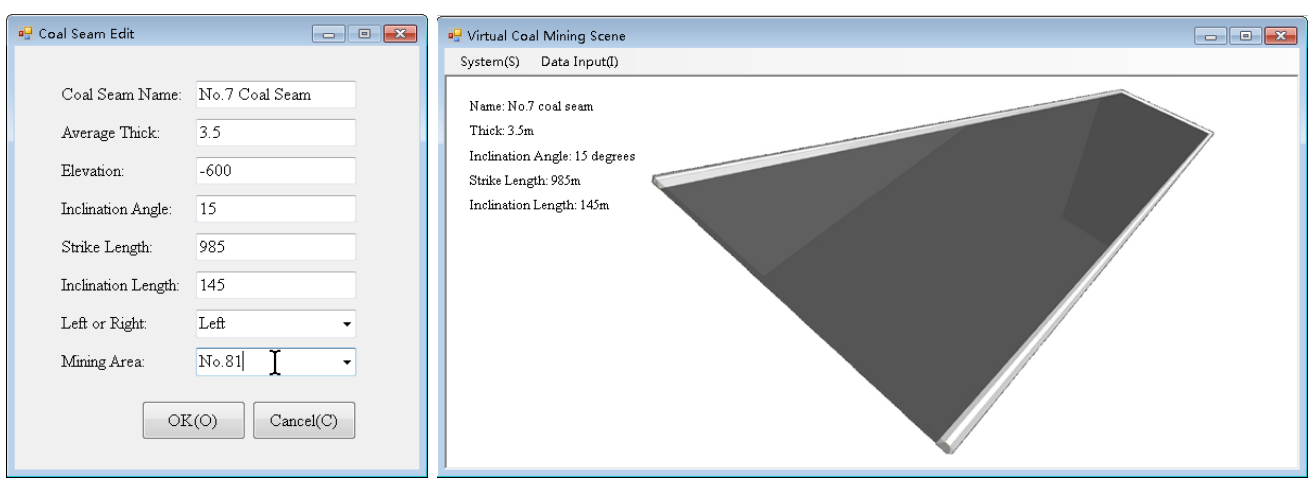

(a) Coal Seam Edit (b) Coal Seam and Coal Laneway Display
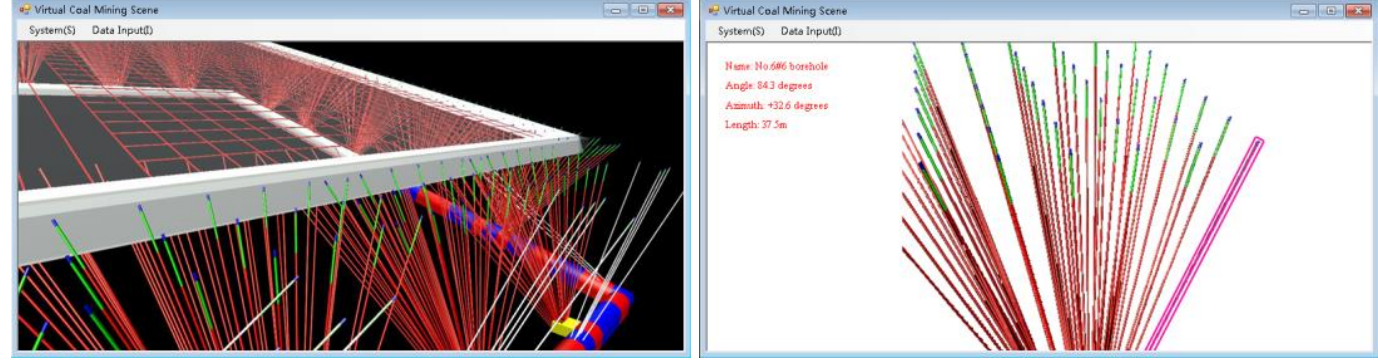

(c) Cross-coal-seam Boreholes Display

(d) Screen Pickup

\section{Figure 9. Application Effect}

\section{Conclusions}

A dynamic and data-driven method for the generation of the virtual coal mining scene was successfully built up and its supported experimental software was developed. The architecture contains two kinds of models and four business layers. From bottom to top, the models are primary models and display models, and the business layers are storage layer, display layer, combination layer and operation layer. Specifically, the following work was finished:

Elaboration of the regional coal gas control technology of the single outburst coal seam. As the most widely used measure for the coal gas pre-drainage of the underground collieries, the construction work of "cross-coal-seam boreholes combined with alongcoal-seam boreholes" was elaborated in detail and selected as the basic case of the study. 
Development of the primary models and display models. The components involved in the construction work of coal gas pre-drainage were disassembled into a number of primary models and stored into the primary library. After setting their parameters, the primary models were transformed into display models and then compose the target virtual scene.

Development of the four business layers. The system business is logically divided into storage layer, display layer, combination layer and operation layer from bottom to top. The storage layer is to store all data of models or the system; the display layer and combination layer is to generate display models by doing transformation on the primary models, and then to build the virtual scene; the operation layer is to respond to the users' instructions such as free roaming or pickup operation. The four layers cooperate to fulfill the entire scene rendering and human-computer interaction businesses.

Finnaly, the data-driven virtual reality system of coal mining process was tested in Qinan colliery of China. Results show that the proposed dynamic generation method of the virtual scene is practicable and efficient. Its application can improve the digitalization and visualization degree of underground coal mining and then prevent the coal gas accident occurrence.

\section{Acknowledgements}

The financial support by University Science and Technology Research Projects of Hebei Province (Grant No.Y2012038) and Heber Provincial Science and Technology Project (Grant No.13453712) is deeply appreciated.

\section{References}

[1] L. Liu and F. B. Zhou, "A comprehensi fe hazard evaluation system for spontaneous combustion of coal in underground mining", International ournal of Coal Geology, vol. 82, (2010), pp. 27-36.

[2] H. F. Wang, Y. P. Cheng, S. J. How, R. Xu an CH. B. Liu, "Application of continuously pressurerelieving technology on long-distance upper protected seam of inclined coal seams", Journal of Mining \& Safety Engineering, vol. 27, no. 2, (2010) pp. 210-214.

[3] S. J. Hou, Y. W. Zhang and Y. P. Cheng, "A computer aided design and simulation system for upward cross-seam boreholes', Journal of Applied Sciences, vol. 13, (2013), pp. 2538-2543.

[4] S. J. Hou and Y W Zhang, "A novel lgorithm for the coverage homogeneity analysis of along-seam boreholes in the coal gas drainage project", Energy Education Science and Technology Part A: Energy Science and Research, vol. 31, no. 3, (2013), pp. 1803-1812.

[5] S. J. Hou, J. Y. Liu and PW. Zhang, "Development of boreholes construction deviation tracking software system: BCD Ttacking", Information Technology Journal, vol. 12, no. 19, (2013), pp. 49184924.

[6] Q. Ma, B. J. Du, J J. Guo and X. Wei, "Building and dynamic generation technology for the virtual equipment basec on Wéb3D”, Electronic Science and Technology, vol. 25, no. 3, (2012), pp. 51-54.

[7] Microsoft Corporation - XNA FrameWork Class Library. Available from: http://msdn.microsoft.com/en-us/ library/ bb203940 (v-XNAGameStudio.31).aspx, (2010).

[8] D. H. Eberly, "3D game engine design: a practical approach to real-time computer graphics (Second Edit on), San Fransisco: Morgan Kaufmann, (2006), pp. 341-358.

[9] Z.J Tian, J. Lei, S. Y. Liu and L. P. Weng, "Problems of safety training for special operation personnel

in coal mines and application of virtual reality technology", China Coal, vol. 41, no. 1, (2015), pp. 104107.

[10] Y. C. Zhang, "Application of virtual reality technology in mine escape simulation training system", Coal Technology, vol. 11, (2013), pp. 274-275.

[11] AIMS Research Unit, School of Chemical, Environmental \& Mining Engineering, University of Nottingham. Virtual Reality Demos. Available from: http://www. Notting ham.ac.uk/aims/VRSite/HTML/Demo.htm, (2015).

[12] Y. Jiang, "Research and implementation 3D virtual reality engine based on the XNA", Ship Electronic Engineering, vol. 33, no. 11, (2013), pp. 86-89.

[13] B Denby and D Schofield, "Role of Virtual Reality in safety training of mine personnel", Mining Engineering, vol. 10, (1999), pp. 59-64.

[14] B. S. Wang, "Study on 3D models and visual technology of the virtual reality system of coal mine", Master thesis of the PLA Information Engineering University, (2006). 
[15] I. B. Shirokov, N. V. Koval and E. V. Koval, "Microwave control system of state change of gaseous atmosphere in mines. Microwave and Telecommunication Technology (CriMiCo)", 2010 20th International Crimean Conference. IEEE, vol. 13, no. 9, (2010), pp. 1081-1082.

[16] J. T. Guo, "Monitoring and supervision system underground coal mine", Coal Technology, vol. 28, no. 6 , (2009), pp. 97-99.

[17] Y. P. Cheng, J. H. Fu and Q. X. Yu, "Development of gas extraction technology in coal mines of China", Journal of Mining \& Safety Engineering, vol. 26, (2009), pp. 127-139.

[18] State Administration of Work Safety. No. (2011)163 instruction, Gas extraction provisional regulations for coal mine. Beijing: China Coal Industry Publishing House, (2011).

[19] State Administration of Work Safety. Coal and gas outburst prevention regulations, 2009. Available from: http://www.chinacourt.org/law/ detail/2009/05/id/136150.shtml, (2014).

[20] Y. P. Cheng and Q. X Yu, "Development of regional gas control technology for Chinese coal mines", Journal of Mining \& Safety Engineering, vol. 24, no. 4, (2007), pp. 383-390.

\section{Authors}
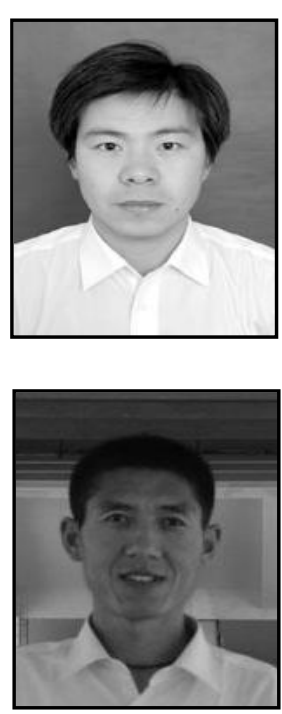

Xianzun Meng, Working as a lecturer of Computer Center at Hebei University of Economics and Business, and studying on the field of industrial software) application. Ph./Fax: +86 031187655672/+86 18603298296. E-mail address: mengxzun@163.com (Xianzun Meng). professor of Computer Center at Hebei University of Economics and Business, and studying on fields of virtual reality technology and industrial software application. Ph./Fax: 486 031 187655672/+86 18132660356. E-mail address: shaojhoy@63.com(Shaojie Hou)
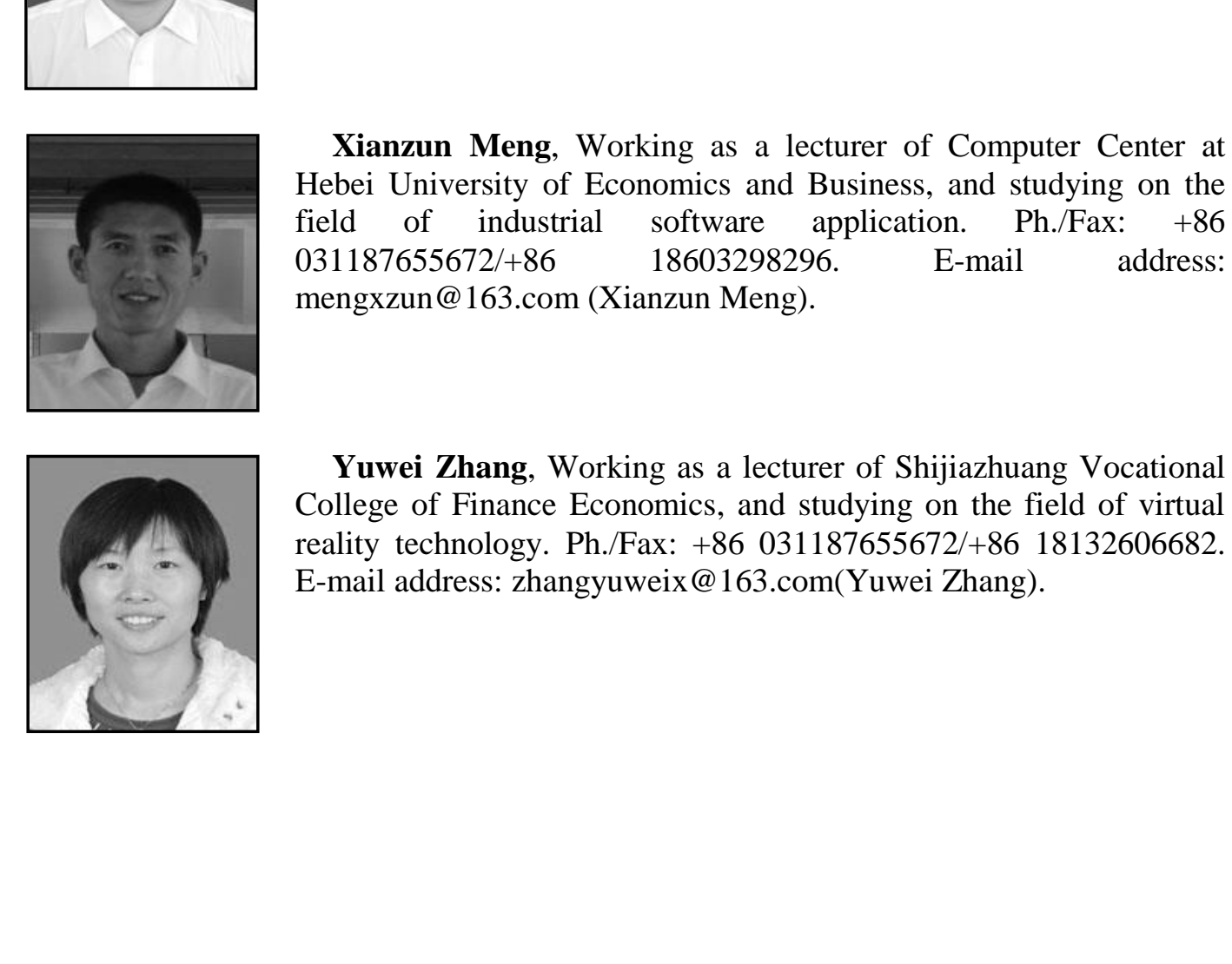